\title{
INTEGRATED OPTICAL CIRCUIT FOR FOCUS AND TRACKING CONTROL ON A MAGNETOOPTICAL DISC
}

\author{
V. LAPRAS, C. CHABROL and J.-B. ALBERTINI \\ LETI-CEAITechnologies Avancées, CENGIDOPT, 17 rue des Martyrs, 38054 Grenoble Cedex 9, France
}

Integrated optics could take the place of existing bulk detection optics in future heads, with the benefits of decreasing size and cost and increasing reliability. Several solutions have been already described [1], [2], [3], employing various technologies and detection principles. In this paper, we present an integrated optical circuit (IOC) combining focus and tracking control (figure 1). This circuit will complete the reading circuit already described in [4] in order to achieve an integrated device with all detection functions (M.O. reading, focus and tracking control).

These circuits are supposed to be used only for detection, not for writing, and they are therefore designed to receive the light reflected back from the M.O. disc. The detection principles are standard ones; focus and tracking control are separated and involve two channels. The reflected beam, coupled into the integrated optical circuit and collimated by the first parabolic mirror (1), is separated in two channels by the first beam splitter (2). The light going through the beam splitter (2) is used for focus control, for which we have chosen the "wax-wane" method. It consists in two "defocused" channel guides (MG1 and MG2) associated with two parabolic mirrors (respectively 5 and 4). The guides have opposite focus shifts, one is located before the theoretical focal point of the associated parabolic mirror, the other is located behind the focal point of the associated parabolic mirror. When the light reflected by each parabolic mirror ( 4 or 5 ) is focused before or behind the intended focus point, the intensity coupled into each guide is not equal. Thus, the difference in intensity at the output of the guides gives a focus error signal. The best way to achieve tracking control is to apply the push-pull method to integrated optics. Then, the role of the IOC is just to separate both sides of the reflected beam. The light reflected by the beam splitter (2), is reflected again by the dual mirrors (6) in order to separate it into two halves and to compare the intensities of each side. The difference between these intensities gives the tracking error signal.

The implemented technology is based on a silicon nitride core between two silica cladding layers ( $\mathrm{Si} / \mathrm{SiO}_{2} / \mathrm{Si}_{3} \mathrm{~N}_{4} / \mathrm{SiO}_{2}$ ). This technology is a low cost technology well adapted for mass production. The optical components of the circuits are made by standard "contact" photolithography and reactive ion etching. The parabolic mirrors are achieved by etching the three layers of the waveguide. They are based on total reflection under high incidence angles. The beam splitters are achieved by etching only the upper cladding layer, so called superstrate, therefore modifying the effective index of the guide. The ratio between transmitted and reflected light is adjusted by fixing the width of the groove. The singlemode channel guides with a $2 \mu \mathrm{m}$ width are achieved in two steps (1/ total etching of the upper cladding layer on both sides of the guide, $2 / 1000 \AA$ silica deposition).

We have characterised these circuits on a static test bench. We use either a mirror or a piece of M.O. disc mounted on a piezo-electric actuator in order to test the focus and tracking control functions. By a modulation of the piezo-electric and with a control of the disc displacement by an interferometric measurement, we have measured the response of our circuits. The oscilloscope screen recordings are presented figure 2 (focus) and figure 3 (tracking). The sensitivity of our focus control circuit is in agreement with the theoretical one which is calculated by means of a "Beam Propagation Method" based numerical tool. The operational range is a little bit smaller than planned $(4 \mu \mathrm{m})$ but it could be adapted by an optimisation of the circuit design. The signal levels are different on each output guide, this is due to technological fluctuations which cause imbalance of the beam splitter (3). Concerning the tracking control, the signals of both outputs have different phases and allow a differential detection of the tracking error signal. The measurement is done by moving the disc perpendicularly to the tracks in order to cross several tracks $(\approx 6)$. 
We have achieved an integrated optical circuit for focus and tracking control. The experimental study has demonstrated that its sensitivity is compatible with the specifications of today's servo circuits. In further investigation, measurements on Philips-IBM 128 MB M.O. drive will be carried out in order to validate this concept in realistic environment.

\section{Acknowledgements}

This work has been partly supported by the Commission of the European Communities under the "ESPRIT 7801" programme.

\section{References}

[1] "TE/TM Mode Splitter with Waveguide Photodetectors" Tami KIHARA SPIE Vol. 1751 (1992)

[2] 'Integrated Optics for Optical Data Storage" Bulent N. KURDI (IBM), ECIO'93

[3] "Integration of Detection Optics for Magnetooptical Disc Pick-up" T. SUHARA, H. ISHIMARU, S. URA, H. NISHIHARA (OSAKA University), The Transactions of the IEICE, Vol. E73, $n^{\circ} I$, Jan. 1990

[4] "Development of a Reading Integrated Optical Circuit for a Magneto-optical Head" V. LAPRAS, P. LABEYE, P. GIDON, European Conference on Integrated Optics, ECIO'93

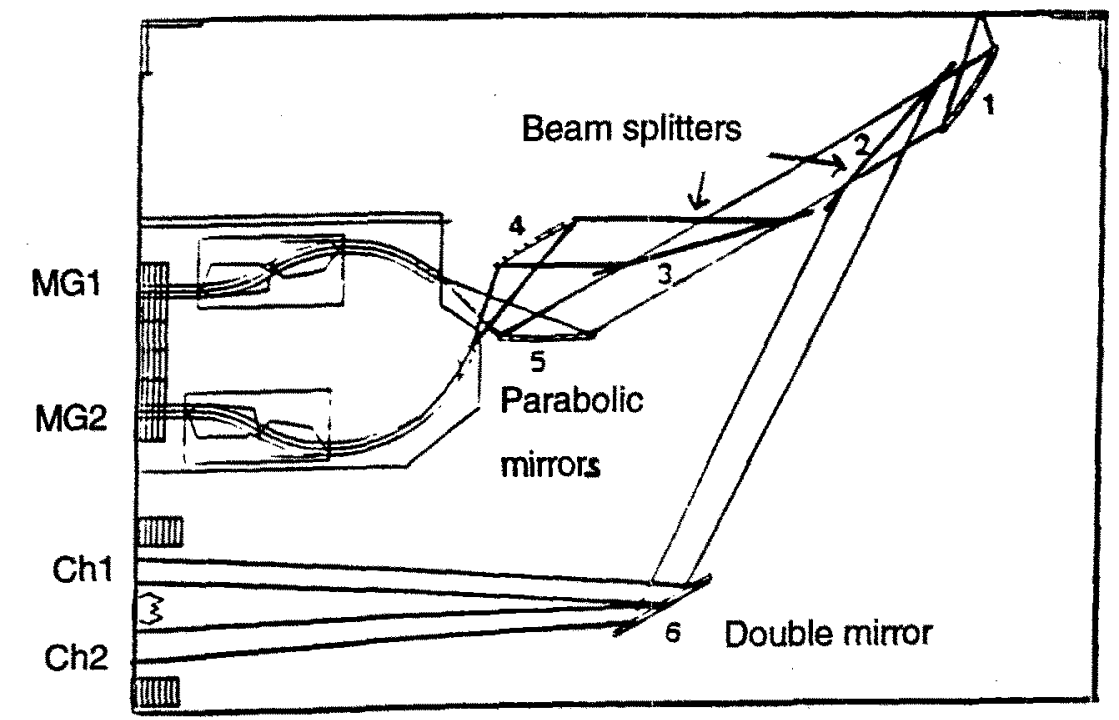

Figure 1 : Schema of the focus and tracking control circuit

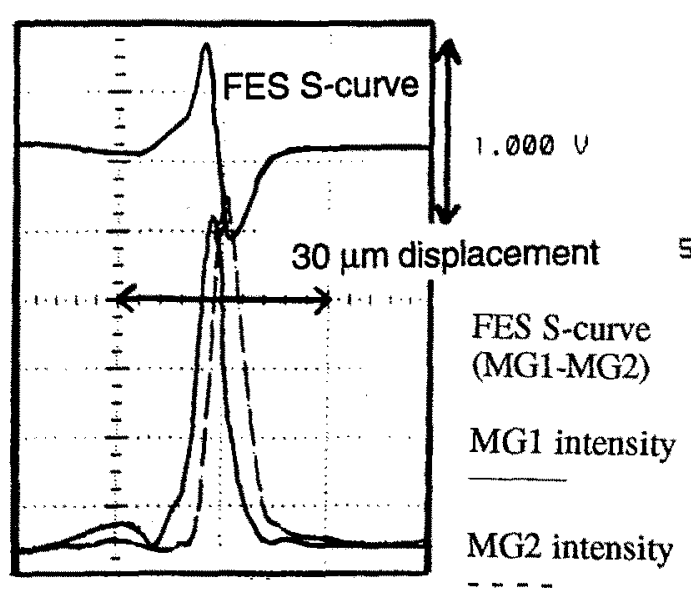

Figure 2 : Focus error signal

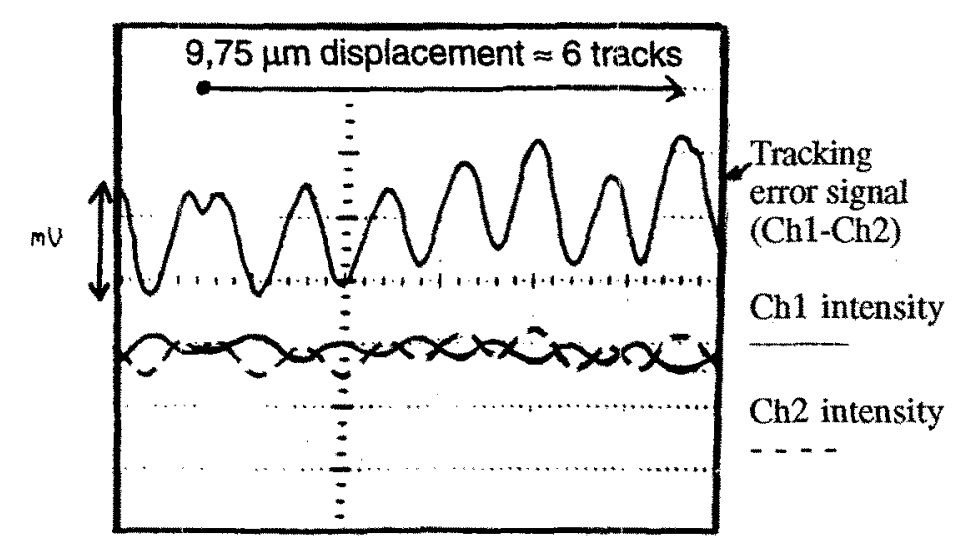

Figure 3 : Tracking error signal 